\title{
Peculiarities of the mathematical modelling of dynamic loading on containers in flat wagons transportation
}

\author{
Oleksij Fomin ${ }^{1}$, Juraj Gerlici², Alyona Lovskaya ${ }^{3}$, Kateryna Kravchenko ${ }^{2, *}$, Oleksii \\ Burlutski ${ }^{4}$, and Vladimir Hauser ${ }^{2}$ \\ ${ }^{1}$ State University of Infrastructure and Technology, Department of Cars and Carriage Facilities, 04071, \\ Kyrylivska str., 9, Kyiv, Ukraine \\ ${ }^{2}$ University of Zilina, Department of transport and handling machines, 01026, Univerzitná 8215/1, \\ Zilina, Slovak Republic \\ ${ }^{3}$ Ukrainian State University of Railway Transport, Department of Wagons, 61050, Feuerbach Square 7, \\ Kharkov, Ukraine \\ ${ }^{4}$ Ukrainian State University of Railway Transport, Department of Mechanics and Machine Design, \\ 61050, Feuerbach Square 7, Kharkov, Ukraine
}

\begin{abstract}
The study deals with a shunting impact of 13-4085 wagon platform to a TK25 tank container. In order to define the loads acting on the container frame, 3D computer models of the 1CC size container is designed, both version - for liquid and dry material transport. The virtual model consider a longitudinal force from the wagon automatic coupling, vertical and horizontal forces from the wagon - containers interaction and also vertical forces of the container fittings. For checking adequacy of the created models, the container acceleration values was obtained in two scenarios. In the first case, when fittings are aligned relative to the fitting spots of a wagon, thus without possibility of relative movement. In the second case, movement within clearance of fitting spots is possible. Mathematical models of dynamic loads on these structures were solved by MathCad. Differential equations were solved by the Runge - Kutta method. Adequacy of the created models was checked with an F-test. On the basis of conducted calculation can be conclude that the hypothesis on the adequacy of the models was not rejected.
\end{abstract}

Keywords: container, dynamics, shunting impact modelling, structure accelerations load, combined transportation

\section{Relevance}

The most popular transport facility used in international combined transportation are containers. A higher efficiency of container transportation along international transport

\footnotetext{
*Corresponding author: kkatherina@ukr.net

Reviewers: Zbigniew Kłos, Josef Soukup
} 
corridors requires development and implementation of the new generation of transport facilities with improved engineering and economical performance.

Objective of the article is improvement of mathematical models and processing of dynamic loads values which influences the carrying structures of containers in operation.

Method. In order to specify values of dynamic loads which influence the carrying structures of the containers placed on flat wagons while shunting impacts occurs and so causes the highest load on their carrying structures in operation, mathematical models of dynamic loads on these structures were created in MathCad. The differential equations were solved by the Runge-Kutta method.

Research of the containers dynamic load was also conducted by computer modelling in CosmosWorks by the Finite Element Method; 3D computer model of the carrying structures of the 1CC container for transportation of liquid and dry freight was created, as well. The model 13-4085 built by the company DniproVahonMash was taken as the basic flat wagon structure. The load model considered a longitudinal force of 3.5 MN acting to the back beam in the area of the flat wagon coupling, vertical and horizontal forces in areas where the flat wagon interacted with containers, and also vertical forces influencing the container fittings. The friction forces in areas where the flat wagon interacted with the containers were taken into account by using the modal damping coefficient.

The adequacy of the designed models was checked with an F-test. The conducted calculation made it possible to conclude that the hypothesis on the adequacy of the models was not rejected.

Findings. The acceleration values, as dynamically loading components influencing containers when the fittings do not move relative to the fitting stops of the flat wagons and when their displacement was possible, were obtained. Analysis of the obtained acceleration values showed that they exceeded the values specified in the normative documents.

Analysis of the obtained results may encourage designing of the new containers generation with the carrying structures with improved technical and economical characteristics.

\section{Nomenclature}

$M_{F L} \quad$ is the carrying structure mass of a flat wagon;

$I_{F L} \quad$ is the inertia moment of a flat wagon relative to the longitudinal axis;

$S_{\mathrm{a}} \quad$ is the value of the longitudinal impact force toward the coupling;

$f_{F R} \quad$ is the peak value of a dry friction force;

$m_{B} \quad$ is the bogie mass;

$I \quad$ is the wheelset inertia moment;

$r \quad$ is the wheel radius in the medium worn state;

$n \quad$ is the number of bogie wheelsets;

$l \quad$ is a half wheelbase of the flat wagon;

$F_{F R} \quad$ is the absolute value of the dry friction force in a spring group;

$k_{1}, k_{2} \quad$ is the spring stiffens of the bogie suspension;

$k \quad$ is the number of oscillation frequencies of the liquid freight;

$m_{i} \quad$ is the mass of a body equivalent to the $i$-th tank container with part of the liquid freight unmovable relative to the tank;

$m_{i j} \quad$ is the mass of the $j$-th pendulum in the $i$-th tank container;

$z_{c i} \quad$ is the height of the tank container load centre;

$c_{i j} \quad$ is the distance between the plane $z_{i}=0$ and the fixation point of the $j$-th pendulum in the $i$-th tank container;

$l_{i j} \quad$ is the length of the $j$-th pendulum; 
$I_{\theta} \quad$ is the reduced inertia moment of the $i$-tank container and liquid freight unmovable relative to the tank;

$I_{i j} \quad$ is the inertia moment of a pendulum;

$x, \varphi, z$ are the coordinates corresponding to the longitudinal, angular (around the longitudinal axis) and vertical movements of a flat wagon;

$x_{i}, \varphi_{i}$ are the coordinates corresponding to longitudinal, angular (around the longitudinal axis) movements of a tank container;

$\xi_{i j} \quad$ is the deviation angle of the $j$-th pendulum off the vertical line.

\section{Introduction}

It is well known that one of the most mobile transportation means used in international combined transportation are containers (Fig. 1) [1-3].

The container interoperability is in a high demand in combined transportation. It requires development and implementation of the new generation containers with improved technical and economical characteristics.

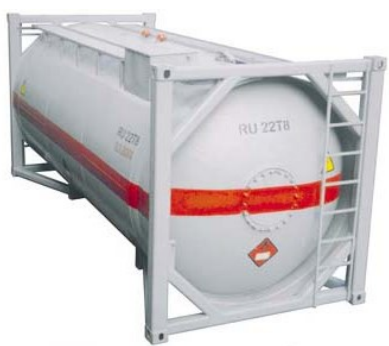

a)

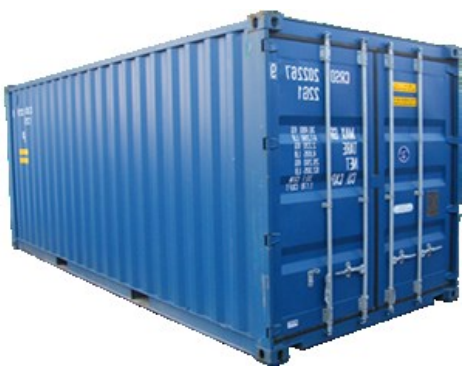

b)

Fig. 1. Containers: a) for liquid freight transport; b) for dry freight transport

\section{The problem statement}

A higher efficiency of transport along international corridors (ITC) requires development and implementation of new transport means generation. The existing mathematical models need optimization for obtaining improved values of the dynamic loads influencing carrying structures of containers in operation. In order to define the loads influence of the containers carrying structures while shunting impacts, the authors used mathematical modelling of dynamic loads on structures in MathCad and computer simulation in CosmosWorks by applying the Finite Element Method. To implement this, 3D computer models of the carrying structures for both, 1CC liquid and dry freight containers were designed. After checking the adequacy of designed models, the acceleration values can be obtained; these values consists of the dynamic loads influencing the containers when fittings do not move relative to the fitting stops of a flat wagon, and also of the load when their displacement is possible. 


\section{Literature review}

The analysis of dynamic loads influencing a tank container placed on a flat wagon while shunting impacts is given in [4].

The accelerations influencing the tank container were defined with consideration of clearances between fitting stops of the flat wagon and fittings of the tank container. The longitudinal force influencing the flat wagon from a hammer car was approximately 2200$2800 \mathrm{kN}$.

The research was conducted on tank containers for transportation of petrol and nitric acid; their gross weights were 21.9 tons and 24.0 tons respectively.

It should be noted that the maximum value of the longitudinal impact force which can influence the loaded flat wagon (including boiler containers) while shunting impacts is 3.5 MN [5]. Thus, in order to get more accurate values of the acceleration influencing the tank container in operation, there is a need for some additional research.

The research of the TK25 tank container capacity and optimization of its carrying structure is given in [6]. The tank container capacity model considers the normative values of loads given in [7]. Research of the carrying structure capacity of a tank container with the improved operational values of loads (displacement of liquid freight, displacement of fittings relative to the fitting stops, etc.) was not conducted.

Problems of designing ideal carrying structures of freight wagon frames and requirements they have to meet at the current stage of the rail transport development are considered in $[8,9]$.

The study does not deal with the designing of ideal containers carrying structures.

\section{Materials and methods}

Analysis of the normative documents for providing operational tank container capacities can be conclude that the highest dynamic loads which influence their carrying structures and fastening units are presented in GOST 31232 "Containers for transportation of dangerous freight. Operational safety requirements" [7]. It is supposed that the structure of a tank container must carry its own inertia forces appearing in operation and while shunting impacts of wagons, including shunting from humps, emergency braking at low speeds as well as under following accelerations: in longitudinal direction $-2 \mathrm{~g}$; in transverse direction $-1 \mathrm{~g}$, in vertical direction $-2 \mathrm{~g}$; under shunting impacts for a loaded container $-4 \mathrm{~g}$; for an empty container (while tests in order to check the accessories) $-5 \mathrm{~g}$.

In order to define operational values of the dynamic loads influencing the carrying structure of a tank container placed on the flat wagon while shunting impacts the mathematical model given in [4] was applied. The model considers that three tank containers were placed on a long-base flat wagon, and the link between them was simulated as elastic with friction, i.e. each container had its own degree of freedom in the vertical plane. It should be noted that tank container transportation also uses framed flat wagons which can carry two containers. Such wagons have a shorter base, and, therefore, lower values of the frame displacement under vertical loads from the containers.

Therefore, the study consider with the 13-4085 flat wagon build at the DniproVahonMash and with the TK25 tank container build at VAT Zarechenskii Zavod Himicheskogo Mashinostroeniia. This is a typical container of ISO 1CC type and is intended for transportation of petroleum products and lubricants, petrol, diesel fuel, soot, solvent naphtha, petroleum solvent and foaming agent.

The diagram of the longitudinal force influencing the flat wagon with containers on is given in Fig. 2. 
The tank container was considered as an additional mass on the flat wagon frame, with additional degree of freedom in the longitudinal direction within the clearances between fitting stops of the flat wagon and fittings of the tank container. Thus, the tank container has its own degree of freedom until the fitting rests against the fitting spot, then the tank container repeats the movement of the flat wagon.
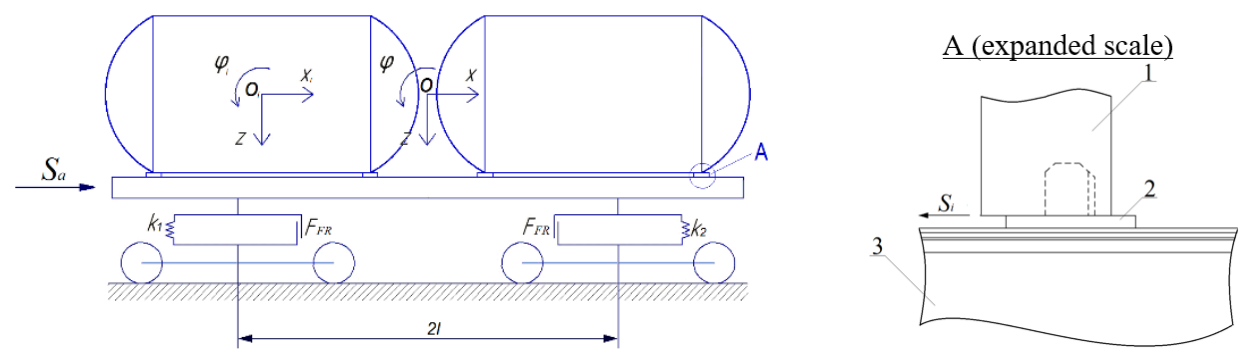

Fig. 2. Diagram of the longitudinal force on the flat wagon with containers: 1 - fitting, 2 - fitting stop, 3 - longitudinal beam of the flat wagon

Connection between the flat wagon frame and fittings of the tank container was simulated as frictional. Besides, the tank containers placed on the flat wagon were taken as those with the equal liquid freight [10].

Mathematical model of described situation is defined by formula (1) to (14).

$$
\begin{aligned}
& M_{F L}^{\prime} \cdot \ddot{x}_{F L}+M_{F L} \cdot h \cdot \ddot{\varphi}_{F L}=S_{a}-\sum_{i=1}^{2} S_{i}, \\
& I_{F L} \cdot \ddot{\varphi}_{F L}+\mathrm{M}_{F L} \cdot h \cdot \ddot{x}_{F L}-g \cdot \varphi_{F L} \cdot \mathrm{M}_{F L} \cdot h=l \cdot F_{F R}\left(\operatorname{sign} \dot{\Delta}_{1}-\operatorname{sign} \dot{\Delta}_{2}\right)+ \\
& +l\left(k_{1} \dot{\Delta}_{1}-k_{2} \dot{\Delta}_{2}\right) \\
& M_{F L} \cdot \ddot{z}_{F L}=k_{1} \cdot \Delta_{1}+k_{2} \cdot \Delta_{2}-F_{F R}\left(\operatorname{sign} \dot{\Delta}_{1}-\operatorname{sign} \dot{\Delta}_{2}\right), \\
& \left(m_{i}+\sum_{j=1}^{k} m_{i j}\right) \cdot \ddot{x}_{i}+\left(m_{i} \cdot z_{c i}+\sum_{j=1}^{k} m_{i j} \cdot c_{i j}\right) \cdot \ddot{\varphi}_{i}-\sum_{j=1}^{k} m_{i j} \cdot l_{i j} \cdot \xi_{i j}=S_{i} \text {, } \\
& \left(I_{\theta i}+\sum_{j=1}^{k} m_{i j} \cdot c_{i j}^{2}\right) \cdot \ddot{\varphi}_{i}+\left(m_{i} \cdot z_{c i}+\sum_{j=1}^{k} m_{i j} \cdot c_{i j}\right) \cdot \ddot{x}_{i}-\sum_{j=1}^{k} m_{i j} \cdot l_{i j} \cdot \xi_{i j}- \\
& -g \cdot\left(m_{i} \cdot z_{c i}+\sum_{j=1}^{k} m_{i j} \cdot c_{i j}\right) \cdot\left(\varphi_{F L}-\varphi_{i}\right)=0 \\
& \left(m_{i}+\sum_{j=1}^{k} m_{i j}\right) \cdot \ddot{z}_{F L}=0 \text {, } \\
& I_{i j} \cdot \ddot{\xi}_{F L}-m_{i j} \cdot l_{i j} \cdot \ddot{x}_{i j}-m_{i j} \cdot c_{i j} \cdot l_{i j} \cdot \ddot{\varphi}_{i j}+g \cdot m_{i j} \cdot l_{i j} \cdot \ddot{\xi}_{i j}=0 \text {, }
\end{aligned}
$$

where 


$$
\begin{gathered}
\mathrm{M}_{F L}^{\prime}=\mathrm{M}_{F L}+2 \cdot m_{B}+\frac{n \cdot I}{r^{2}} ; \Delta_{1}=z_{F L}-l \cdot \varphi_{F L} ; \Delta_{2}=z_{F L}+l \cdot \varphi_{F L}, \\
S_{i}=f_{F R} \cdot \operatorname{sign} \cdot\left(x_{F L}-x_{i}\right)^{\prime} .
\end{gathered}
$$

And $x_{i}<30 \mathrm{~mm}[4,11]$, if $x_{i} \geq 30 \mathrm{~mm}$, then $x_{i}=x$. Therefore,

$$
\begin{aligned}
& M_{F L}^{\prime} \cdot \ddot{x}_{F L}+M_{F L} \cdot h \cdot \ddot{\varphi}_{F L}=S_{a} \text {, } \\
& I_{F L} \cdot \ddot{\varphi}_{F L}+M_{F L} \cdot h \cdot \ddot{x}_{F L}-g \cdot \varphi_{F L} \cdot M_{F L} \cdot h=l \cdot F_{F R}\left(\operatorname{sign} \dot{\Delta}_{1}-\operatorname{sign} \dot{\Delta}_{2}\right)+ \\
& +l\left(k_{1} \dot{\Delta}_{1}-k_{2} \dot{\Delta}_{2}\right) \\
& M_{F L} \cdot \ddot{z}_{F L}=k_{1} \cdot \Delta_{1}+k_{2} \cdot \Delta_{2}-F_{F R}\left(\operatorname{sign} \dot{\Delta}_{1}-\operatorname{sign} \dot{\Delta}_{2}\right), \\
& \left(m_{i}+\sum_{j=1}^{k} m_{i j}\right) \cdot \ddot{x}_{F L}+\left(m_{i} \cdot z_{c i}+\sum_{j=1}^{k} m_{i j} \cdot c_{i j}\right) \cdot \ddot{\varphi}_{F L}-\sum_{j=1}^{k} m_{i j} \cdot l_{i j} \cdot \ddot{\xi}_{i j}=0 \text {, } \\
& \left(I_{\theta i}+\sum_{j=1}^{k} m_{i j} \cdot c_{i j}^{2}\right) \cdot \ddot{\varphi}_{F L}+\left(m_{i} \cdot z_{c i}+\sum_{j=1}^{k} m_{i j} \cdot c_{i j}\right) \cdot \ddot{x}_{F L}+\sum_{j=1}^{k} m_{i j} \cdot c_{i j} \cdot l_{i j} \cdot \ddot{\xi}_{i j}- \\
& -g \cdot\left(m_{i} \cdot z_{c i}+\sum_{j=1}^{k} m_{i j} \cdot c_{i j}\right) \cdot \varphi_{F L}=0 \\
& \left(m_{i}+\sum_{j=1}^{k} m_{i j}\right) \cdot \ddot{z}_{F L}=0 \text {, } \\
& I_{i j} \cdot \ddot{\xi}_{i j}-m_{i j} \cdot l_{i j} \cdot \ddot{x}_{i j}-m_{i j} \cdot c_{i j} \cdot l_{i j} \cdot \ddot{\varphi}_{F L}+g \cdot m_{i j} \cdot l_{i j} \cdot \ddot{\xi}_{i j}=0 .
\end{aligned}
$$

The vertical displacements of the tank container relative to the flat wagon frame were not considered. The displacement of the liquid freight relative to the walls of the boiler was taken into account. The liquid freight movement was described as a set of mathematical pendulums [4]. The value of the longitudinal impact influencing the flat wagon was taken as $3.5 \mathrm{MN}$. The hydrodynamic characteristics of the liquid freight were defined by the method presented in [12]. Petrol was taken as the liquid freight. On the base of the calculations conducted for the case of the maximum possible loading on the container tank in accordance with [13], the values $m_{i j} \approx 6.8 \mathrm{t}$ and $I_{i j} \approx 6.8 \mathrm{t} \cdot \mathrm{m}^{2}$ were obtained.

The differential equations were solved by the Runge-Kutta method in MathCad.

Results of the research allowed the authors to conclude that without gaps between fitting stops of the flat wagon and fittings of the tank container, the acceleration influencing the carrying structure of the tank container was approximately $40 \mathrm{~m} / \mathrm{s}^{2}$. The maximum acceleration was obtained for a gap between a fitting stop and a fitting of $30 \mathrm{~mm}$, the acceleration being about $300 \mathrm{~m} / \mathrm{s}^{2}$.

In modelling dynamic loading for a dry freight container the above mentioned mathematical model was reduced to [14]:

$$
M_{F L}^{\prime} \cdot \ddot{x}_{F L}+M_{F L} \cdot h \cdot \ddot{\varphi}_{F L}=S_{a}-\sum_{i=1}^{2} S_{i},
$$




$$
\begin{gathered}
I_{F L} \cdot \ddot{\varphi}_{F L}+M_{F L} \cdot h \cdot \ddot{x}_{F L}-g \cdot \varphi_{F L} \cdot M_{F L} \cdot h= \\
=l \cdot F_{F R}\left(\operatorname{sign} \dot{\Delta}_{1}-\operatorname{sign} \dot{\Delta}_{2}\right)+l\left(k_{1} \cdot \Delta_{1}-k_{2} \cdot \dot{\Delta}_{2}\right), \\
M_{F L} \cdot \ddot{z}_{F L}=k_{1} \cdot \Delta_{1}+k_{2} \cdot \Delta_{2}-F_{F R}\left(\operatorname{sign} \dot{\Delta}_{1}-\operatorname{sign} \dot{\Delta}_{2}\right), \\
m_{i} \cdot \ddot{x}_{i}+\left(m_{i} \cdot z_{c i}\right) \cdot \ddot{\varphi}_{i}=S_{i}, \\
I_{i} \cdot \ddot{\varphi}_{i}+\left(m_{i} \cdot z_{c i}\right) \cdot \ddot{x}_{i}-g \cdot\left(m_{i} \cdot z_{c i}\right) \cdot\left(\varphi_{F L}-\varphi_{i}\right)=0, \\
m_{i} \cdot \ddot{z}_{F L}=0 .
\end{gathered}
$$

The vertical displacements of the container relative to the flat wagon frame were not considered.

The results of the research allowed the authors to conclude that with gaps between fittings of the container and fitting stops of the flat wagon the accelerations influencing the carrying structure was approximately $110 \mathrm{~m} / \mathrm{s}^{2}$.

In order to define the acceleration influencing the container placed on the flat wagon while shunting impacts without gaps between fittings and fitting spots, the mathematical model was reduced to:

$$
\begin{gathered}
\mathrm{M}_{F L}^{\prime} \cdot \ddot{x}_{F L}+\mathrm{M}_{F L} \cdot h \cdot \ddot{\varphi}_{F L}=S_{\mathrm{a}} \\
I_{F L} \cdot \ddot{\varphi}_{F L}+\mathrm{M}_{F L} \cdot h \cdot \ddot{x}_{F L}-g \cdot \varphi_{F L} \cdot \mathrm{M}_{F L} \cdot h= \\
=l \cdot F_{F R}\left(\operatorname{sign} \dot{\Delta}_{1}-\operatorname{sign} \dot{\Delta}_{2}\right)+l\left(k_{1} \cdot \Delta_{1}-k_{2} \cdot \dot{\Delta}_{2}\right), \\
M_{F L} \cdot \ddot{z}_{F L}=k_{1} \cdot \Delta_{1}+k_{2} \cdot \Delta_{2}-F_{F R}\left(\operatorname{sign} \dot{\Delta}_{1}-\operatorname{sign} \dot{\Delta}_{2}\right), \\
m_{i} \cdot \ddot{\mathrm{x}}_{F L}+\left(m_{i} \cdot z_{c i}\right) \cdot \ddot{\varphi}_{F L}=0, \\
I_{i} \cdot \ddot{\varphi}_{F L}+\left(m_{i} \cdot z_{c i}\right) \cdot \ddot{\mathrm{x}}_{F L}-g \cdot\left(m_{i} \cdot z_{c i}\right) \cdot \varphi_{F L}=0, \\
m_{i} \cdot \ddot{z}_{F L}=0,
\end{gathered}
$$

thus, it disregarded the friction forces between fitting spots and fittings and the inertia forces appearing when the container moved relative to the flat wagon frame. The calculation showed that the maximum acceleration influencing the container was nearly $50 \mathrm{~m} / \mathrm{s}^{2}$.

\section{Experiments}

In order to check acceleration values obtained by computer modelling of dynamics for the loaded container wagon under acting force of $3.5 \mathrm{MN}$ to the back beam of the coupling in longitudinal direction, problem was conducted in CosmosWorks (version 2015) [15]. The calculation was made by the Finite Element Method. The finite element model was built with spatial isoparametrical tetrahedrons.

The model considered both, the longitudinal force and the vertical force acting to the flat wagon in areas, where the containers rested upon fitting stops. It was considered that the container was influenced by the vertical force in areas where the fitting rested upon the fitting spots. To consider friction forces in the areas of interaction between the flat wagon and the containers, the coefficient of modal damping was applied.

The $09 \Gamma 2 \mathrm{C}$ steel of the strength limit $\sigma_{\mathrm{B}}=490 \mathrm{MPa}$ and the yield strength $\sigma_{\mathrm{T}}=345 \mathrm{MPa}$ was taken as material for the carrying structure of the flat wagon and the containers. 


\section{Findings}

Numerical values of accelerations influencing the carrying capacity of the containers placed on the flat wagon while shunting impacts are given in Table 1.

Table 1. The numerical values of the acceleration influencing the carrying structures of the containers placed on the flat wagon while shunting impacts

\begin{tabular}{|c|c|c|}
\hline \multirow{2}{*}{ Container type } & \multicolumn{2}{|c|}{ Acceleration, $\mathrm{m} / \mathrm{s}^{2}$} \\
\cline { 2 - 3 } & $\begin{array}{c}\text { without the clearance between } \\
\text { fitting and fitting spot }\end{array}$ & $\begin{array}{c}\text { with the clearance between } \\
\text { fitting and fitting spot }\end{array}$ \\
\hline Liquid freight container & 40 & 300 \\
\hline Dry freight container & 50 & 110 \\
\hline
\end{tabular}

The adequacy of the designed models was checked by an F-test. The conducted calculation made it possible to conclude that the hypothesis on adequacy of the models was not rejected.

\section{Discussion}

The conducted research showed that accelerations influencing containers placed on the flat wagon while shunting impacts considerably exceeded the normative values, a possible movement of fittings relative to fitting spots being taken into account [7]. Therefore, there is a need to improve the normative documents by adding boundary values of the maximum acceleration, potentially acting on containers placed on the flat wagon while shunting impacts, and also to consider specified values of dynamic loads already in the stage of wagon and its facilities designing.

\section{Conclusions}

The conducted research led to the following conclusions:

1. The research of the dynamic loads on containers placed on the flat wagon provided specified values of accelerations influencing the containers in operation. It has been proved that accelerations influencing a container while shunting impacts greatly depended on gaps between fittings and fitting spots and exceeded the normative values [7].

2. The safety requirements should be provided by limiting movements of containers placed on the flat wagon relative to their frames while shunting impacts.

3. Consideration of specified values of accelerations influencing containers in operation is required for designing new generation of containers.

The study was a state funded research: "Building conceptually new module freight wagons as the basis for Ukraine to compete in the rail transportation market and the machine building sector" (State Registration \#0117U000564), and "Recommendations against operational damages of freight wagon carrying systems by example of the TH 4-201 thermos car built by Dessau (Germany)" (State Registration \#0116U007820), where the authors were executive officer, responsible officer and supervisor.

\section{References}

1. T.V. Butko, A.V. Prokhorchenko, A. Kyman, Formalization of the technology of arranging tactical group trains. Eastern-European Journal of Enterprise Technologies. 3 (76), 38-43 (2015) 
2. N. Gorbunov, R. Domin, M. Kovtanec, K. Kravchenko, The multifunctional energy efficient method of cohesion control in the "wheel-braking pad-rail" system. Prace Naukowe Politechniki Warszawskiej - Transport. Międzynarodowej Konferencji Naukowej TRANSPORT XXI WIEKU, Arłamów. 114-126 (2016)

3. V. Hauser, O. Nozhenko, K. Kravchenko, M. Loulová, J. Gerlici, T. Lack, Impact of Three Axle Boxes Bogie to the Tram Behavior When Passing Curved Track, Procedia Engineering 192, 295-300 (2017)

4. G. I. Bogomaz, D.D. Mehov, O.P. Pilipchenko, Yu.G. Chernomashentseva, Load of tank containers located on a railway platform, when striking a coupler, Collection of scientific works «Dynamics and control of movement of mechanical systems». Kyiv: ANU, Institute of Technical Mechanics. 87-95 (1992) [In Russian]

5. Norms for the calculation and designing of railway cars of the IGS track $1520 \mathrm{~mm}$ (nonself-propelled). GosNIIV. VNIIZhT, 319 (1996) [In Russian]

6. A.O. Lovska, O.M. Melnichuk, O.V. Fomin, Improvement of tank-containers for transportation of petroleum products by introducing into their supporting structures round tubes. Railway transport of Ukraine 1, 40 - 44 (2015) [In Ukrainian]

7. Containers for the transport of dangerous goods. Requirements for operational safety. GOST 31232. Minsk: "Belarusian State Institute of Standardization and Certification". 6 (2005) [In Russian]

8. O.V. Fomin, Improvement of upper bundling of side wall of gondola cars of 12-9745 model. Scientific and technical journal «Metallurgical and Mining Industry 1, 45-48 (2015)

9. M. Kelrykh, O. Fomin, Perspective directions of planning carrying systems of gondolas. Scientific and technical journal «Metallurgical and Mining Industry». No. 6, 64-67 (2014)

10. A.O. Lovska, Investigation of the strength of the load-bearing structure of a containercistern located on a platform wagon with a shunting collision. Collection of scientific works. Kiev: SUIT 28, 90-98 (2016) [In Ukrainian]

11. Rules for the placement and fastening of goods in wagons and containers when transporting them by railways of the gauge of $1520 \mathrm{~mm}$ of the SMGS participating countries. Part 1. General provisions. OSJD, 681 (2012) [In Russian]

12. Yu.P. Krivovyazyuk, Evaluation of the equivalent loading of four-axle rail tank cars with liquid cargo of different density for longitudinal impact. Thesis PhD: Dnepropetrovsk, 157 (1986) [In Russian]

13. Rules for the transport of dangerous goods. To the agreement on international railway cargo communication 3. - OSJD, 531 (2011) [In Russian]

14. A. Lovska, A. Rybin, The study of dynamic load on a wagon-platform at a shunting collision. Eastern-European Journal of Enterprise Technologies 3, 4-8. (2016)

15. A.A. Alyamovskiy, SolidWorks/COSMOSWorks 2006 - 2007. Engineering analysis by the finite element method, (Series "Designing"), 784 (2007) [In Russian] 